\title{
LARGE EDDY SIMULATION, TURBULENT TRANSPORT AND THE RENORMALIZATION GROUP*
}

\author{
J. GLIMM ${ }^{\dagger} \S$, B. PLOHR $\ddagger$, AND D. H. SHARP A $^{\ddagger}$
}

\begin{abstract}
In large eddy simulations, the Reynolds averages of nonlinear terms are not directly computable in terms of the resolved variables and require a closure hypothesis or model, known as a subgrid scale term. Inspired by the renormalization group (RNG), we introduce an expansion for the unclosed terms, carried out explicitly to all orders. In leading order, this expansion defines subgrid scale unclosed terms, which we relate to the dynamic subgrid scale closure models. The expansion, which generalizes the Leonard stress for closure analysis, suggests a systematic higher order determination of the model coefficients.

The RNG point of view sheds light on the nonuniqueness of the infinite Reynolds number limit. For the mixing of $N$ species, we see an $N+1$ parameter family of infinite Reynolds number solutions labeled by dimensionless parameters of the limiting Euler equations, in a manner intrinsic to the RNG itself. Large eddy simulations, with their Leonard stress and dynamic subgrid models, break this nonuniqueness and predict unique model coefficients on the basis of theory. In this sense large eddy simulations go beyond the RNG methodology, which does not in general predict model coefficients.
\end{abstract}

Key words. LES, renormalization group, subgrid scale models

AMS Subject Classification: 76F65, 76F35, 82B28

\section{Introduction.}

1.1. Turbulence and large eddy simulations. Turbulence is one of the major unsolved problems of classical physics, a view attributed to Heisenberg, among others. Turbulence is an instability of fluid flow which occurs at high Reynolds numbers $\operatorname{Re}=V L / \nu$, where $V$ and $L$ are representative velocities and lengths and $\nu$ is the kinematic anisotropic viscosity. Many turbulent flows, including ones arising in oceanography, atmospheric sciences, aeronautics, astrophysics and chemical processing occur at elevated, nearly infinite Reynolds numbers.

Turbulence is a strong coupling theory with no natural length scales. For this reason numerical solutions of turbulent flow, necessarily cut off at some grid level, encounter difficulties in the coupling between the resolved (grid level) data and the unresolved (subgrid level) data. Because of the strong coupling, scale invariant nature of subgrid scale turbulence, methods to treat this effect on the resolved scales have been introduced. Large Eddy Simulation (LES) is such a numerical method in which some, but not all, of the turbulent scales are resolved, and subgrid scale models emulate the influence of the unresolved on the resolved scales.

The strong coupling problem originates in the nonlinearity of the compressible Navier-Stokes equations, which govern the turbulent flow field. The averaging oper-

\footnotetext{
*April 25, 2022

$\dagger$ Department of Applied Mathematics and Statistics, Stony Brook University, Stony Brook, NY 11794-3600, USA and Computational Science Center, Brookhaven National Laboratory, Upton, NY 11793-6000, USA

‡Theoretical Division, Los Alamos National Laboratory, Los Alamos, NM, 87545, USA

$\S$ Supported in part by Leland Stanford Junior University 2175022040367A (subaward with DOE as prime sponsor), Army Research Office W911NF0910306. This manuscript has been co-authored by Brookhaven Science Associates, LLC, under Contract No. DE-AC02-98CH1-886 with the U.S. Department of Energy. The United States Government retains, and the publisher, by accepting this article for publication, acknowledges, a world-wide license to publish or reproduce the published form of this manuscript, or allow others to do so, for the United States Government purposes. Los Alamos National Laboratory Preprint Number LA-UR-12-26149

॥ Supported by Los Alamos National Laboratory (retired).
} 
ation used to define grid level quantities suitable for numerical computation, when applied to nonlinear terms in the Navier-Stokes equations, leads to new unknown flow quantities, called subgrid scale (SGS) terms. Supplying approximations to the SGS terms is a process known as closure. The averaged nonlinear terms, before approximation, are called unclosed.

1.2. RNG applied to LES. In the limit $\operatorname{Re} \rightarrow \infty$, the theory simplifies and scaling laws apply, the most famous of which is that of Kolmogorov [19. The renormalization group (RNG) is a method for the systematic study of scaling laws. The RNG framework is explained in 28, with the goal of finding an expression for the unclosed terms in a LES mentioned briefly. Our expansion accomplishes this goal, using the RNG expansion but not an RNG fixed point. Relevant to the present work, we mention our own studies of turbulent mixing [22, 24, 17, 29]. We also mention our related $\mathrm{K} 41$ based existence theorem for $L^{p}$ solutions of the incompressible constant density Euler equations [4].

The study of SGS terms is central to this paper. Each closed SGS approximation term is regarded as a product of a coefficient and a solution functional of specified form. We call the latter a model. The RNG expansion derived here, through the dynamic method of coefficient selection, uniquely specifies the coefficient once the model is given. This determination is based on properties of the resolved scales, connected to analogous properties of the unresolved scales by an asymptotic assumption. Thus the coefficient is determined by theory, leaving the model selection open as a subject for research. The search for alternate frameworks for the choice of SGS terms is an active topic of research, which we do not attempt to review here, but we do discuss problems which arise if the SGS models are omitted completely, as commonly occurs when the Implicit Large Eddy Simulation (ILES) method is used in practice.

Our first main result is a closed form expansion for the unclosed terms in the Reynolds averaged equations, founded on RNG ideas, with (for a finite expansion) a closed form remainder. RNG methods, by themselves, do not predict the equation coefficients, so that dynamic LES methods, which do, are an extension of the RNG methodology. At leading order, this expansion is a step in the derivation of closures for the quadratic SGS terms, coinciding with the derivation of the Leonard stress. The leading order term of our expansion is related to the Clark model [5]. Due to stability problems with the Clark model, which arise because the SGS terms are not definite, the exact closures, even in leading order, are further approximated, most commonly by gradient diffusion models in a Smagorinsky tradition. The second main contribution in this paper, of a more speculative nature, consists of observations concerning the nonuniqueness of the $\operatorname{Re} \rightarrow \infty$ limit, a phenomenon that is naturally understood within the RNG framework. We also identify numerical nonuniqueness as an issue for verification methodology. Here we also make contact with the ILES method, in which explicit SGS terms are replaced by algorithmic details, resulting in nonunique (effective, numerically or algorithmically driven) SGS terms and nonuniqueness of the solution.

We begin with the second result, nonuniqueness, as the terminology needed for the rest of the paper is introduced in this manner. In the RNG line of reasoning, the Euler equations, as a limit of the Navier-Stokes equations, arise as an RNG fixed point. We have postulated [24, 17] that for the mixing of $N$ species, the high Re limit point is not unique; rather, it is chosen from an $N+1$ parameter family of limit points, labeled by points $T \in \mathcal{T}$, with $\mathcal{T}$ being the $(N+1)$-dimensional space of transport coefficient ratios for the Navier-Stokes equation, namely the $N-1$ indepen- 
dent dimensionless mass diffusivities (Schmidt numbers), the dimensionless thermal diffusivity (the Prandtl number), and the dimensionless ratio of the anisotropic and isotropic viscosities. In the case of multi-temperature thermodynamics, $\mathcal{T}$ is $2 \mathrm{~N}$ dimensional, the increase resulting from $N-1$ new Prandtl numbers. Each sequence $\operatorname{Re} \rightarrow \infty$ is accompanied by a sequence $T(\operatorname{Re}) \in \mathcal{T}$, which we assume to be convergent: $T(\mathrm{Re}) \rightarrow T(\mathrm{Re}=\infty)$. Dimensional transport coefficients are denoted as $t$. In the limit $\operatorname{Re} \rightarrow \infty$ as we define it, $t \rightarrow 0$, but other choices of the limit $\operatorname{Re} \rightarrow \infty$ are possible, as we illustrate below. Further comments on nonuniqueness are given in 8

As a simple illustration of the nonuniqueness of the RNG limit, consider two different physically motivated pictures. In case $\mathrm{A}$, as a model of turbulent combustion, the thermal diffusion is molecular in nature and enhanced by turbulent mixing. For this limit, we keep fixed the dimensionless Prandtl number $\operatorname{Pr}=\nu / \kappa$, where $\kappa$ is the thermal diffusivity and $\nu$ is the kinematic anisotropic viscosity, to preserve the dimensionless aspects of the thermal mixing processes; the other dimensionless transport coefficients are also fixed in the same manner. In case B, we assume a thermal process unrelated to turbulent mixing (such as radiation), for which we want to keep $\kappa$ constant so that $\operatorname{Pr} \rightarrow 0$ as $\operatorname{Re} \rightarrow \infty$. The limiting equations in the two cases $\mathrm{A}$ and $\mathrm{B}$ do not coincide. For case A, the limiting equations are the Euler equations, with all transport coefficients set to zero, and for case B, the limiting equations include a thermal diffusion term in the energy equation.

The full $N+1$ parameter space of RNG limit points is realized by alternate paths in $\mathcal{T}$, taken as $\operatorname{Re} \rightarrow \infty$. Alternate paths may originate from alternate choices for the subgrid scale models, or from alternate physical modeling, leading to alternate settings of the laminar transport coefficients $t(\mathrm{Re})$, as indicated above (cases A and B).

Related to this physics-level nonuniqueness is a numerical nonuniqueness. Different numerical algorithms applied to an identical problem yield apparently converged solutions with significant qualitative differences [10, 27, 29]. Not only are the SGS terms which select among the $N+1$ parameter family of solutions commonly omitted or treated implicitly as an aspect of the algorithm itself, but also numerical truncation errors contribute at a comparable order and also participate in the selection of the limit from among the $N+1$ parameter family of possible solutions. We observe that nonuniqueness has been demonstrated mathematically for some time [37.

1.3. Three RNG steps. The RNG has three basic operations:

1. an integration or coarse-graining step, which partially solves the equation in mapping the unknown solution from a fine to a coarser grid;

2. a remapping or rescaling step, which changes the length scales so that the unknown length scale of the coarser grid is always fixed;

3. a re-parametrization step, in which certain solution parameters (the coefficients of the "relevant" terms) are assigned new, or updated, values to assure continued agreement with parameters that define the observed solutions. This agreement is obtained from measurements at the length scale of the coarse grid.

We propose to label the relevant variables by the $N+1$ parameters of $\mathcal{T}$; see additional discussion in 88 . For each parameter of $\mathcal{T}$, we need an observable, defined on the coarse grid, to determine that parameter. To establish a bridge to the LES terminology, we call these observables models.

We apply the RNG construction to estimate subgrid scale terms associated with a grid $\mathcal{M}_{n}$. On this grid some terms (namely, the averages of the nonlinear terms in the Navier-Stokes equations) are unresolved, in the sense that they fail to be functions 
only of the averaged primitive variables. The averaged primitive variables are constant on each cell of the grid $\mathcal{M}_{n}$.

The RNG step 1 is an integration, i.e., a coarse graining step. This map of solutions induces a map of equations, namely the equations satisfied by the mesh averages of the solutions. The map of equations is straightforward as far as linear equation terms are concerned. (See Secs. 2 and 3.) But the map leads to unresolved (unclosed) terms when applied to nonlinear terms in the equation. These unclosed (as far as $\mathcal{M}_{n}$ is concerned) terms are identified as polynomials in first difference operators defined on $\mathcal{M}_{n+1}$. We iterate on step 1 , with step 2 removed. In this way, we start the coarse graining, in successive RNG iterations, from $\mathcal{M}_{n+2}, \cdots, \mathcal{M}_{n+k}, \cdots$. This construction of unclosed terms for equations on $\mathcal{M}_{n}$ yields a product of polynomials in first difference operators on each of $\mathcal{M}_{n+1}, \cdots, \mathcal{M}_{n+k}, \cdots$.

In this mapping the SGS terms, the expressions unresolved at some mesh level $\mathcal{M}_{n}$ are partially integrated, meaning that their values on a finer mesh $\mathcal{M}_{n+k}$ are determined, up to expressions unresolved on this finer mesh. The RNG step 1, as implemented in this paper, is to express these terms, unresolved on $\mathcal{M}_{n}$, on successively finer grids $\mathcal{M}_{n+k}$.

In our treatment of step 2, we depart from conventional RNG methodology. We omit the remap step 2. For any finite number of steps, the remap is an isomorphism, and so its presence or absence is moot. However in the limit $k \rightarrow \infty$, there is a difference. The conventionally remapped RNG analysis would place the LES data at infinitely remote (spatial infinity) wave numbers to achieve the integration of a truly scale invariant problem. In the LES framework, we do not wish to eliminate the problem data in this manner, and so we forgo the remap and the RNG fixed point, while obtaining a RNG based series expansion for the unclosed $\mathcal{M}_{n}$ SGS terms. To emphasize this distinction, we refer to the limit we obtain as an RNG limit point (but not an RNG fixed point).

According to step 3, at each RNG step the equation parameters (the RNG relevant variables) should be set equal to the physical parameter values for that length scale. This must occur at the grid level $\mathcal{M}_{n+k}$.

We define turbulent and total dimensional transport parameters, the latter denoted $t_{\text {total }}$. We take the step 3 requirement to mean that the $N+1$ total (laminar plus turbulent) transport coefficients $t_{\text {total }} \in \mathcal{T}$ should be set at each RNG step. We could as well set the dimensionless transport coefficients. For this purpose, we follow convention and define $T_{\text {total }}=\nu_{\text {total }} / t_{\text {total }}$ as the dimensionless transport parameters. Sequences with different limit points $T_{\text {total }}(R e=\infty)$ for the dimensionless total transport should generate different infinite Re solutions of the Navier-Stokes or Euler equations.

As conventionally applied, for example to quantum field theory, the relevant variables (particle masses and coupling constants) are predicted neither by the RNG fixed point nor by the quantum field theory itself. The mass of the proton, a fundamental particle in strong coupling quantum field theory, is derived from an extended theory of quarks, in which the proton is not a fundamental particle but rather a derived object. More directly comparable is quantum electrodynamics. In principle, an exact integration of the RNG equation would give the observed (finite length scale) mass of the electron as a multiple of the bare (zero length scale) mass, but the bare mass is not known. We also compare to quantum chromodynamics (QCD), which shares with turbulence theory the property that the coupling constant (the turbulent viscosity, for turbulence theory) becomes smaller at short length scales. This property of QCD 
is called asymptotic freedom. In turbulence theory, a similar exact integration of the RNG defines the turbulent diffusion coefficient as observed at finite length scales in terms of the same coefficient as observed at zero length scales. However, in contrast to quantum electrodynamics, this "bare" diffusion coefficient is generally accepted to be zero, thus is known. Moreover, for turbulent diffusion, the RNG integration is dominated by its low order perturbation term, and thus is effectively determined perturbatively, as with dynamic SGS models. Thus the dynamic LES turbulence theory of the infinite Re limit goes beyond the quantum field theory RNG in predicting its own coefficients.

1.4. Models and RNG relevant parameters. To set parameters according to RNG step 3 at the length scale of $\mathcal{M}_{n+k}$, we need observables at this same length scale, and with $N+1$ parameters to be set, we need to specify $N+1$ observables, each an observable relative to the mesh $\mathcal{M}_{n+k}$. In other words, the observables should be functions of the averaged primitive variables on the mesh $\mathcal{M}_{n}$. These observables constitute the model for the unobserved SGS terms, and RNG step 3 supplies the (missing) coefficients for the model; i.e., it sets the coefficients for turbulent transport at each RNG step. In conventional RNG applications, the model coefficients are set by comparison to experimental data. Here they are set by theory. This coefficient specification is equivalent to specifying a point $T_{\text {total }} \in \mathcal{T}$ at each RNG step.

The coefficients for the SGS terms have the form $\nu_{\text {total }} / \mathrm{Sc}_{\text {total }}, \nu_{\text {total }} / \mathrm{Pr}_{\text {total }}$ and a similar ratio for the isotropic viscosity coefficient.

Dynamic LES is a single expansion step, $k=1$, of the RNG expansion. For this step, it contains the same steps 1 and 3 as the RNG and two additional steps:

4. Conventionally, we replace the continuous time but space discretized quantities of the RNG steps 1-3 with a time discretization as well. Thus the SGS terms are defined as the average over a time step of the cell face averages introduced below. To define a closed LES algorithm, we replace these unclosed SGS terms with the corresponding models multiplied by their coefficients as defined by step 3 in the numerical solution of the equations on the grid $\mathcal{M}_{n}$.

5. Model coefficients are determined by a theoretical analysis from the mesh level solutions to the governing equations.

In the RNG formalism, we label expansion terms at each order as irrelevant, relevant, or nonrenormalizable according to whether they become smaller, remain constant or grow as the renormalization map is iterated, and we consider the effects of finer grids as refinements of the grid $\mathcal{M}_{n}$. There should be no nonrenormalizable parameters. We anticipate $N+1$ relevant ones, namely the $N+1$ coefficients of the models, interpreted as dimensionless total transport coefficients.

Settings of the relevant parameters follow from the dynamic SGS analysis [31, a version of which is repeated here. This method specifies an Re-dependent path $T_{\text {turbulent }}(\operatorname{Re}) \in \mathcal{T}$. These coefficients are combined with the laminar ones $T(\operatorname{Re}) \in \mathcal{T}$ through a formula $t_{\text {total }}=t_{\text {turbulent }}+t_{\text {laminar }}$ and together define $T_{\text {total }}(\operatorname{Re}) \in \mathcal{T}$ which uniquely specifies the LES method. The dynamic SGS method assumes a functional form for the model, and given that, derives the coefficients. Variations in this formalism can be based on alternate formulations for the model. The dynamic SGS method determines the coefficients of the model directly from the solutions at grid level $\mathcal{M}_{n}$ of the governing equations, and seems to leave little room for argument regarding the coefficients, given the model.

1.5. Outline. Our formalism is developed in $\$ 2$ and illustrated for compressible mixing in 93 , The expansions for SGS terms are developed in 4 . The leading order 
expansion terms are discussed in 96 , with the relation to the Leonard stress given in 45. In 97, we explore the possible role of numerical issues in modifying or selecting the limit point. A concluding discussion is found in 48

2. Averaging Procedure. The Reynolds-averaged equations associated with a system of conservation laws are obtained through application of a Reynolds averaging operator, such as ensemble averaging. Similarly, the equations that are discretized in an LES method arise from application of an integral operator, viz., convolution with a filter. Both a Reynolds averaging operator and convolution with a filter commute with spatial and temporal derivatives.

We prefer instead to use the averaging operator defined by volume integration over the cells of a spatial grid. Although the time derivative commutes such volume averaging, spatial derivatives do not. We compensate by viewing the cell averages of the solution of the conservation laws as solving a finite volume approximation to the system.

2.1. Discretized equations. Let us write the system of conservation laws under consideration as

$$
\frac{\partial U}{\partial t}+\frac{\partial F_{i}(U)}{\partial x_{i}}=0
$$

where repeated tensor indices are summed. Here $U$ denotes the vector of densities of conserved quantities (e.g., mass, momentum, energy, and species). To this system we apply the cell averaging operator associated with a polygonal finite-volume mesh $\mathcal{M}$. For a cell $\mathcal{C}$ of $\mathcal{M}$, let $\bar{a}^{\mathcal{C}}$ denote the volume integral over $\mathcal{C}$ of the quantity a divided by the volume $V(\mathcal{C})$ of $\mathcal{C}$. By the divergence theorem, the volume integral over $\mathcal{C}$ of the divergence of the flux $F_{i}(U)$ equals the surface integral of the outward normal component of the flux over the boundary $\partial \mathcal{C}$. This boundary consists of the faces of the cell. If $f \subset \partial \mathcal{C}$ is a face of $\mathcal{C}$, let $\bar{b}^{f}$ denote the surface integral over $f$ of the quantity $b$ divided by the surface area $A(f)$ of $f$. Then averaging Eq. (2.1) over the cell $\mathcal{C}$ yields the semi-discrete evolution equation

$$
\frac{\mathrm{d} \bar{U}^{\mathcal{C}}}{\mathrm{d} t}+\sum_{\left\{f \subset \partial \mathcal{C}: f \text { a face of } \mathcal{M}_{\backslash}\right\}} \frac{A(f)}{V(\mathcal{C})} n_{i}^{\mathcal{C}, f}{\overline{F_{i}(U)}}^{f}=0,
$$

where $n_{i}^{\mathcal{C}, f}$ is the unit normal to face $f$ pointing outward from $\mathcal{C}$.

The face-averaged flux ${\overline{F_{i}(U)}}^{f}$ is constructed as follows. First, we write

$$
{\overline{F_{i}(U)}}^{f}=F_{i}\left(\bar{U}^{f}\right)+F_{i}^{f, \mathrm{SGS}}(U) .
$$

This equation defines the SGS flux $F_{i}^{f, \text { SGS }}(U)$. Explicit formulae for the SGS flux components for compressible mixing are given in $\$ 3$, an expansion for each SGS term, which is the central result of the present paper, is developed in 4 Second, $F_{i}\left(\bar{U}^{f}\right)$ is related to cell-averaged conserved quantities through a numerical scheme, such as

$$
n_{i}^{\mathcal{C}, f} F_{i}\left(\bar{U}^{f}\right)=\mathcal{F}\left(\bar{U}^{\mathcal{C}}, \bar{U}^{\mathcal{C}^{\prime}}, n^{\mathcal{C}, f}\right)+\mathcal{E}^{\mathcal{C}, f}(U),
$$

$\mathcal{C}^{\prime}$ being the cell sharing face $f$ with $\mathcal{C}$. Here $\mathcal{F}$ is the numerical flux for a conservative, consistent finite-volume scheme and $\mathcal{E}^{\mathcal{C}, f}$ is its truncation error, discussed in 97 . 
2.2. Projection operators. To be more concrete, we focus on cubic meshes. That is, we take the physical domain to be $\mathcal{D}=[0,1]^{D}$, the $D$-dimensional unit cube with periodic boundary conditions, and we let $\mathcal{M}_{n}$ be the cubic mesh with a corner at the origin that divides $\mathcal{D}$ into equal sized cells, each with edge lengths $2^{-n}$. Thus $\mathcal{M}_{n+1}, \mathcal{M}_{n+2}, \ldots$ are nested refinements of the base mesh $\mathcal{M}_{n}$, which remains fixed throughout the discussion.

Let $E_{n}$ be the operation of averaging over faces of the grid $\mathcal{M}_{n}$. Applied to a function $a$ defined on $\mathcal{F}_{n}$ (the union of the faces of $\mathcal{M}_{n}$ ), this operation yields a piecewise-constant function $E_{n} a$ that is constant over each face, the constant value for face $f$ being its average $\bar{a}^{f}$ over the face. On the Hilbert space $\mathcal{H}_{n}=L^{2}\left(\mathcal{F}_{n}, \mathrm{~d}^{D-1} x\right)$, the operator $E_{n}$ is an orthogonal projection onto the subspace $E_{n} \mathcal{H}_{n}$ comprising such piecewise-constant functions. In fact for face $f$,

$$
\bar{a}^{f}=\frac{\int_{f} a \mathrm{~d}^{D-1} x}{\int_{f} \mathrm{~d}^{D-1} x}=\frac{\left\langle 1_{f}, a\right\rangle}{\left\langle 1_{f}, 1_{f}\right\rangle},
$$

where $1_{f}$ is the characteristic function of $f$, which takes the value 1 on $f$ and zero elsewhere, and the brackets $\langle\cdot, \cdot\rangle$ denote the usual inner product on $\mathcal{H}_{n}$. Therefore

$$
E_{n} a=\sum_{\left\{f \subset \mathcal{F}_{n}: f \text { a face of } \mathcal{M}_{\backslash}\right\}} \bar{a}^{f} 1_{f}=\sum_{\left\{f \subset \mathcal{F}_{n}: f \text { a face of } \mathcal{M}_{\backslash}\right\}} \frac{1_{f}\left\langle 1_{f}, a\right\rangle}{\left\langle 1_{f}, 1_{f}\right\rangle} .
$$

Let $F_{n}=I-E_{n}$ denote the complementary projection operator. When the choice of mesh is unambiguous, we write $\bar{a}$ in place of the mean $E_{n} a$ and $a^{\prime}=a-\bar{a}$ in place of the fluctuation $F_{n} a$.

In addition to the simple averaging operator $E_{n}$, the mass density-weighted, or Favre, averaging operator $\widetilde{E}_{n}$ is useful. We assume that $\rho$ has sufficient regularity to allow its restriction to the faces of $\mathcal{M}_{n}$ and, as restricted, to being locally integrable. On the Hilbert space $\widetilde{\mathcal{H}}_{n}=L^{2}\left(\mathcal{F}_{n}, \rho \mathrm{d}^{D-1} x\right)$, the operator $\widetilde{E}_{n}$ is likewise an orthogonal projection onto the subspace $\widetilde{E}_{n} \widetilde{\mathcal{H}}_{n}$. For face $f$

$$
\widetilde{a}^{f}=\frac{\overline{\rho a}^{f}}{\bar{\rho}^{f}}=\frac{\int_{f} a \rho \mathrm{d}^{D-1} x}{\int_{f} \rho \mathrm{d}^{D-1} x}=\frac{\left\langle 1_{f}, a\right\rangle_{\rho}}{\left\langle 1_{f}, 1_{f}\right\rangle_{\rho}},
$$

where the notation $\langle\cdot, \cdot\rangle_{\rho}$ stands for the usual inner product on $\widetilde{\mathcal{H}}_{n}$. Hence

$$
\widetilde{E}_{n} a=\sum_{f \subset \mathcal{F}_{n}} \frac{1_{f}\left\langle 1_{f}, a\right\rangle_{\rho}}{\left\langle 1_{f}, 1_{f}\right\rangle_{\rho}}
$$

We also let $\widetilde{F}_{n}=I-\widetilde{E}_{n}$ and, when unambiguous, denote the Favre mean $\widetilde{E}_{n} a$ by $\widetilde{a}$ and the Favre fluctuation $\widetilde{F}_{n} a$ by $a^{\prime \prime}=a-\widetilde{a}$.

For $k=1,2, \ldots$, the projection operator $\widetilde{E}_{n+k}$ on $\widetilde{\mathcal{H}}_{n+k}=L^{2}\left(\mathcal{F}_{n+k}, \rho \mathrm{d}^{D-1} x\right)$ is defined similarly. However, we shall regard $\widetilde{E}_{n+k}$ instead as the operator on $\widetilde{\mathcal{H}}_{n}$ that acts in the following way on $a \in \widetilde{\mathcal{H}}_{n}$ : first extend $a$ to all of the faces of $\mathcal{M}_{n+k}$, setting it to zero except on the faces of $\mathcal{M}_{n}$; next apply $\widetilde{E}_{n+k}$; and finally restrict the result to the faces of $\mathcal{M}_{n}$, obtaining $\widetilde{E}_{n+k} a \in \widetilde{\mathcal{H}}_{n}$. The operator $\widetilde{E}_{n+k}$ so defined is a projection operator on $\widetilde{\mathcal{H}}_{n}$. Thus $\widetilde{E}_{n+k} \widetilde{\mathcal{H}}_{n}$ consists of square-integrable functions defined on $\mathcal{F}_{n}$ that are constant on each face of $\mathcal{M}_{n+k}$ contained within a face of $\mathcal{M}_{n}$. 
We note an operator identity that will be useful:

$$
\widetilde{E}_{n}=\widetilde{E}_{n} \widetilde{E}_{n+1} \text {. }
$$

Also, because $\widetilde{E}_{n}$ is a projection operator given by Eq. (2.8),

$$
\left\langle 1_{f}, a \widetilde{E_{n}} b\right\rangle_{\rho}=\left\langle\widetilde{E_{n}}\left(1_{f} a\right), \widetilde{E_{n}} b\right\rangle_{\rho}=\left\langle 1_{f},\left(\widetilde{E_{n}} a\right) \widetilde{E_{n}} b\right\rangle_{\rho},
$$

so that

$$
\widetilde{E}_{n}\left[a \widetilde{E}_{n} b\right]=\widetilde{E}_{n}\left[\left(\widetilde{E}_{n} a\right) \widetilde{E}_{n} b\right] .
$$

3. Averaged Equations for Compressible Mixing. To illustrate the SGS flux defined by Eq. (2.3), we describe it in detail for the system of conservation laws governing the compressible mixing of two gases.

The state of an ideal mixture of two polytropic gases is characterized by the field variables $\rho, v_{i}, T$ and $\psi$, which denote the mass density, particle velocity vector, temperature and mass fraction, respectively. The mixture has pressure $p=\rho R T$, where $R=\psi R_{1}+(1-\psi) R_{2}$, and specific internal energy $e=c_{v} T$, where $c_{v}=$ $\psi c_{v, 1}+(1-\psi) c_{v, 2}$. Here $R_{\alpha}=N_{A} k_{B} / M_{\alpha}$ and $c_{v, \alpha}$ are constants for $\alpha=1,2$. The specific total energy is denoted $E=\frac{1}{2} v_{\ell} v_{\ell}+e$. The specific enthalpy of the mixture is $h=e+p / \rho=c_{p} T$ with $c_{p}=c_{v}+R$, and the individual specific enthalpies for the two gases are $h_{\alpha}=c_{p, \alpha} T$, where $c_{p, \alpha}=c_{v, \alpha}+R_{\alpha}$.

The laminar transport coefficients for momentum, heat, and mass are the kinematic anisotropic and isotropic viscosities, $\nu$ and $\nu_{\mathrm{i}}$, thermal diffusivity $\kappa=\nu / \operatorname{Pr}$ and mass diffusivity $D=\nu / \mathrm{Sc}$, where Pr and Sc denote the Prandtl and Schmidt parameters. The viscous stress tensor, heat flux vector, and diffusive mass flux vector are

$$
\begin{aligned}
\sigma_{i j}^{\mathrm{v}} & =\rho \nu_{\mathrm{i}} \frac{\partial v_{\ell}}{\partial x_{\ell}} \delta_{i j}+\rho \nu\left(\frac{\partial v_{i}}{\partial x_{j}}+\frac{\partial v_{j}}{\partial x_{i}}-\frac{2}{3} \frac{\partial v_{\ell}}{\partial x_{\ell}} \delta_{i j}\right), \\
q_{i} & =-\rho \kappa c_{p} \frac{\partial T}{\partial x_{i}}, \\
j_{i} & =-\rho D \frac{\partial \psi}{\partial x_{i}} .
\end{aligned}
$$

For simplicity, $\rho \nu, \rho \nu_{\mathrm{i}}, \rho \kappa$ and $\rho D$ (hence $\nu / \nu_{\mathrm{i}}$, Pr and Sc) are assumed to be constant.

The cell-averaged laws of conservation of mass, momentum, energy and species, which govern the averaged field variables $\bar{\rho}, \widetilde{v_{i}}, \widetilde{T}$ and $\widetilde{\psi}$, are

$$
\begin{aligned}
& \frac{\partial \bar{\rho}}{\partial t}+\frac{\partial \bar{\rho} \widetilde{v_{i}}}{\partial x_{i}}=0, \\
& \frac{\partial \bar{\rho} \widetilde{v_{j}}}{\partial t}+\frac{\partial\left(\bar{\rho} \widetilde{v_{i}} \widetilde{v_{j}}+\bar{p} \delta_{i j}\right)}{\partial x_{i}}=\frac{\partial \overline{\sigma_{i j}^{\mathrm{v}}}}{\partial x_{i}}-\frac{\partial \tau_{i j}}{\partial x_{i}}, \\
& \frac{\partial \bar{\rho} \widetilde{E}}{\partial t}+\frac{\partial(\bar{\rho} \widetilde{E}+\bar{p}) \widetilde{v_{i}}}{\partial x_{i}}=\frac{\partial \overline{\sigma_{i j}^{\mathrm{v}}} \widetilde{v_{j}}}{\partial x_{i}}-\frac{\partial \overline{q_{i}}}{\partial x_{i}}-\frac{\partial\left(\widetilde{h_{1}}-\widetilde{h_{2}}\right) \overline{j_{i}}}{\partial x_{i}} \\
& \quad-\frac{\partial \tau_{i j} \widetilde{v_{j}}}{\partial x_{i}}-\frac{\partial q_{i}^{(h)}}{\partial x_{i}}-\frac{\partial q_{i}^{(h \psi)}}{\partial x_{i}}-\frac{\partial q_{i}^{(k)}}{\partial x_{i}}-\frac{\partial q_{i}^{(\mathrm{v})}}{\partial x_{i}}, \\
& \frac{\partial \bar{\rho} \widetilde{\psi}}{\partial t}+\frac{\partial \bar{\rho} \widetilde{\psi} \widetilde{v_{i}}}{\partial x_{i}}=-\frac{\partial \overline{j_{i}}}{\partial x_{i}}-\frac{\partial q_{i}^{(\psi)}}{\partial x_{i}} .
\end{aligned}
$$


For clarity, we have made the formal replacement (which is actually an identity in the sense of distribution derivatives)

$$
\sum_{\{f \subset \partial \mathcal{C}: f \text { is a face of } \mathcal{M} \backslash\}} \frac{A(f)}{V(\mathcal{C})} n_{i}^{\mathcal{C}, f} \mapsto \frac{\partial}{\partial x_{i}}
$$

in Eq. (2.2) and omitted the $\mathcal{C}$ and $f$ indications on the averaging operators. (An average of a field variable appearing within a time derivative is a cell average, whereas within a spatial derivative it is a face average.)

Appearing in Eqs. (3.4)-(3.7) are the SGS flux components $\tau_{i j}, q_{i}^{(h)}, q_{i}^{(h \psi)}, q_{i}^{(k)}$, $q_{i}^{(\mathrm{v})}$ and $q_{i}^{(\psi)}$, which are defined by

$$
\begin{aligned}
\tau_{i j} & =\bar{\rho}\left(\widetilde{v_{i} v_{j}}-\widetilde{v_{i}} \widetilde{v_{j}}\right)=\bar{\rho} \widetilde{v_{i}^{\prime \prime} v_{j}^{\prime \prime}}, \\
q_{i}^{(h)} & =\bar{\rho}\left(\widetilde{h v_{i}}-\widetilde{h} \widetilde{v_{i}}\right)=\bar{\rho} \widetilde{c_{p}} \widetilde{T^{\prime \prime} v_{i}^{\prime \prime}}+\bar{\rho}\left(c_{p, 1}-c_{p, 2}\right)\left(\widetilde{T} \widetilde{\psi^{\prime \prime} v_{i}^{\prime \prime}}+\widetilde{\psi^{\prime \prime} T^{\prime \prime} v_{i}^{\prime \prime}}\right), \\
q_{i}^{(h \psi)} & \left.=\overline{\left(h_{1}-h_{2}\right) j_{i}}-\widetilde{\left(h_{1}\right.}-\widetilde{h_{2}}\right) \overline{j_{i}}=\left(c_{p, 1}-c_{p, 2}\right)\left(\overline{T^{\prime \prime}} \overline{j_{i}}+\overline{T^{\prime} j_{i}^{\prime}}\right), \\
q_{i}^{(k)} & =\frac{1}{2} \bar{\rho}\left(\widetilde{v_{\ell} v_{\ell} v_{i}}-2 \widetilde{v_{\ell}} \widetilde{v_{\ell} v_{i}}-\widetilde{v_{\ell} v_{\ell}} \widetilde{v_{i}}+2 \widetilde{v_{\ell}} \widetilde{v_{\ell}} \widetilde{v_{i}}\right)=\frac{1}{2} \bar{\rho} \widetilde{v_{\ell}^{\prime \prime} v_{\ell}^{\prime \prime} v_{i}^{\prime \prime}}, \\
q_{i}^{(\mathrm{v})} & \left.=-\overline{\left(\overline{\sigma_{i j}^{\mathrm{v}} v_{j}}\right.}-\overline{\sigma_{i j}^{\mathrm{v}}} \widetilde{v_{j}}\right)=-\overline{\sigma_{i j}^{\mathrm{v}}} \overline{v_{j}^{\prime \prime}}-\overline{\left(\sigma_{i j}^{\mathrm{v}}\right)^{\prime} v_{j}^{\prime}}, \\
q_{i}^{(\psi)} & =\bar{\rho}\left(\widetilde{\psi v_{i}}-\widetilde{\psi} \widetilde{v_{i}}\right)=\bar{\rho} \widetilde{\psi^{\prime \prime} v_{i}^{\prime \prime}} .
\end{aligned}
$$

Here we have taken advantage of the properties $\overline{a^{\prime}}=0$ and $\widetilde{a^{\prime \prime}}=0$ of cell and face averaging (but not convolution with a filter).

A related SGS quantity, the fluctuation kinetic energy $k$ defined by $\bar{\rho} k=\frac{1}{2} \tau_{\ell \ell}=$ $\frac{1}{2} \bar{\rho} \widetilde{v_{\ell}^{\prime \prime} v_{\ell}^{\prime \prime}}$, arises in the formula $\widetilde{E}=\frac{1}{2} \widetilde{v_{\ell}} \widetilde{v_{\ell}}+\widetilde{e}+k$. The Favre-averaged specific internal energy $\widetilde{e}$ is viewed as $\widetilde{c_{v}} \widetilde{T}$ plus the SGS term $\widetilde{c_{v} T}-\widetilde{c_{v}} \widetilde{T}=\left(c_{v, 1}-c_{v, 2}\right) \widetilde{\psi^{\prime \prime} T^{\prime \prime}}$; and the averaged pressure $\bar{p}=\bar{\rho} \widetilde{R T}$ occurring in the conservation laws is treated similarly. Also, in $\overline{j_{i}}=-\rho D \partial \bar{\psi} / \partial x_{i}$, the variable $\bar{\psi}$ should be replaced by $\widetilde{\psi}$ plus the SGS term $\overline{\psi^{\prime \prime}}$; similarly, the SGS terms $\overline{v_{\ell}^{\prime \prime}}$ and $\overline{T^{\prime \prime}}$ enter into $\overline{\sigma_{i j}^{\mathrm{v}}}$ and $\overline{q_{i}}$, respectively. Notice that a quantity such as $\overline{\psi^{\prime \prime}}$ is equivalently written as $\bar{\rho}\left(\widetilde{\left.\rho^{-1}\right)^{\prime \prime}} \psi^{\prime \prime}\right.$.

Thus we see that an SGS term for the compressible mixing of two gases, under the stated modeling assumptions, involves either a covariance $\operatorname{cov}(a, b)=\overline{a^{\prime} b^{\prime}}$, a Favre covariance $\widetilde{\operatorname{cov}}(a, b)=\widetilde{a^{\prime \prime} b^{\prime \prime}}$, or a third-order Favre cumulant $\widetilde{\operatorname{cum}}(a, b, c)=\widetilde{a^{\prime \prime} b^{\prime \prime} c^{\prime \prime}}$, where $a, b$ and $c$ are each one of $\rho^{-1}, v_{i}, T$ or $\psi$ or their spatial derivatives.

4. Expansions for SGS Terms. The flux appearing in the discretized conservation laws (2.2) is decomposed, via Eq. (2.3), into a function of $\bar{U}=E_{n} U$ plus the SGS flux. A function of $E_{n} U$ is said to be resolved on $\mathcal{M}_{n}$. In this section we develop expansions for SGS terms involving quantities that are resolved on successively finer grids.

4.1. Leonard SGS term. A familiar SGS term is the Reynolds stress tensor $\tau_{i j}^{(n)}$, where

$$
\tau_{i j}^{(n)}=\overline{\rho v_{i} v_{j}}-\overline{\rho v_{i}} \overline{\rho v_{j}} / \bar{\rho} .
$$

The superscript $n$ indicates that the averaging occurs on the grid $\mathcal{M}_{n}$. Consider the corresponding tensor $\tau_{i j}^{(n-1)}=\widehat{\rho v_{i} v_{j}}-\widehat{\rho v_{i}} \widehat{\rho v_{j}} / \widehat{\rho}$ on the once-coarsened grid $\mathcal{M}_{n-1}$, 
where the caret denotes averaging for $\mathcal{M}_{n-1}$, and compare it with $\tau_{i j}^{(n)}$, as averaged onto $\mathcal{M}_{n-1}$. The Germano identity [13] is that the difference $\tau_{i j}^{(n-1)}-\widehat{\tau_{i j}^{(n)}}$ between these two $\mathcal{M}_{n}$-unresolved SGS terms reduces to the Leonard stress tensor [21, 31, 26]

$$
\mathcal{L}_{i j}^{(n-1)}=\widehat{\bar{\rho} \tilde{v}_{i} \tilde{v}_{j}}-\widehat{\bar{\rho} \tilde{v}_{i}} \widehat{\bar{\rho} \tilde{v}_{j}} / \widehat{\bar{\rho}}
$$

which is resolved on $\mathcal{M}_{n}$. The reduction occurs because of the cancellation of the two terms, $\widehat{\rho v_{i} v_{j}}$ and $\widehat{\overline{\rho v_{i} v_{j}}}$, that are not $\mathcal{M}_{n}$-resolved,

4.2. Generalized Leonard SGS term. More generally, consider an SGS term in the form of a Favre covariance

$$
\widetilde{\operatorname{cov}}_{n}(a, b)=\widetilde{a^{\prime \prime} b^{\prime \prime}}=\widetilde{a b^{\prime \prime}}=\widetilde{E}_{n}\left[a \widetilde{F}_{n} b\right]
$$

of the quantities $a$ and $b$ with respect to the mesh $\mathcal{M}_{n}$. (The ordinary covariance $\operatorname{cov}_{n}(a, b)=\overline{a^{\prime} b^{\prime}}$ is included as a special case.) By analogy, we define the corresponding Leonard SGS term to be

$$
\widetilde{\mathcal{L}}_{n}(a, b)=\widetilde{\operatorname{cov}}_{n}(a, b)-\widetilde{E}_{n} \widetilde{\operatorname{cov}}_{n+1}(a, b) .
$$

As we now demonstrate, this quantity is $\mathcal{M}_{n+1}$-resolved provided that $\rho a$ and $\rho b$ are components of $U$.

Using the identity (4.3) on levels $n$ and $n+1$, along with the operator identity (2.9) and the definitions $\widetilde{F}_{n}=I-\widetilde{E}_{n}$ and $\widetilde{F}_{n+1}=I-\widetilde{E}_{n+1}$, we see that

$$
\begin{aligned}
\widetilde{\mathcal{L}}_{n}(a, b) & =\widetilde{E}_{n}\left[a \widetilde{F}_{n} b\right]-\widetilde{E}_{n} \widetilde{E}_{n+1}\left[a \widetilde{F}_{n+1} b\right] \\
& =\widetilde{E}_{n}\left[a\left(\widetilde{E}_{n+1}-\widetilde{E}_{n}\right) b\right] .
\end{aligned}
$$

By identity (2.9) again along with Eq. (2.11) at levels $n$ and $n+1$,

$$
\widetilde{\mathcal{L}}_{n}(a, b)=\widetilde{E}_{n}\left[\left(\widetilde{E}_{n+1} a\right) \widetilde{E}_{n+1} b\right]-\widetilde{E}_{n}\left[\left(\widetilde{E}_{n} a\right) \widetilde{E}_{n} b\right] .
$$

Finally, according to identity (2.9), an $\mathcal{M}_{n}$-resolved quantity is also $\mathcal{M}_{n+1}$-resolved. Hence $\widetilde{\mathcal{L}}_{n}(a, b)$ is $\mathcal{M}_{n+1}$-resolved.

4.3. Expansion for a covariance. Next we develop an expansion for the Favre covariance $\widetilde{\operatorname{cov}}_{n}(a, b)=\widetilde{a^{\prime \prime} b^{\prime \prime}}$. By definition of the generalized Leonard SGS term,

$$
\widetilde{\operatorname{cov}}_{n}(a, b)=\widetilde{\mathcal{L}}_{n}(a, b)+\widetilde{E}_{n} \widetilde{\operatorname{cov}}_{n+1}(a, b) .
$$

By induction on $n$, we see that

$$
\widetilde{\operatorname{cov}}_{n}(a, b)=\widetilde{E}_{n} \sum_{j=0}^{J} \widetilde{\mathcal{L}}_{n+j}(a, b)+\widetilde{E}_{n} \widetilde{\operatorname{cov}}_{n+J+1}(a, b)
$$

The sum involving Leonard terms, which we denote by $\widetilde{\operatorname{cov}}_{n, J}(a, b)$, is resolved on the grid $\mathcal{M}_{n+J+1}$. The remainder is denoted by $R_{n, J}(a, b)$. 
4.4. Expansion for a third-order cumulant. Some of the SGS terms in system (3.4) -(3.7) involve third-order cumulants, such as $\psi^{\prime \prime} T^{\prime \prime} v_{i}^{\prime \prime}$ and $v_{\ell}^{\prime \prime} v_{\ell}^{\prime \prime} v_{i}^{\prime \prime}$. The presence of fluctuations indicates that these terms are not $\mathcal{M}_{n}$-resolved; and because they are not covariances, they are not handled by the methods of 4.3 . Our procedure is to expand each fluctuation factor as an average plus a fluctuation relative to $\mathcal{M}_{n+1}$, and after organizing and simplifying the result, we iterate and generate the expansion with remainder to all orders.

The expansion step is to replace each of the $\widetilde{F}_{n}$ operators in the general third-order cumulant,

$$
\widetilde{\operatorname{cum}}_{n}(a, b, c)=\widetilde{a^{\prime \prime} b^{\prime \prime} c^{\prime \prime}}=\widetilde{E}_{n}\left[\left(\widetilde{F}_{n} a\right)\left(\widetilde{F}_{n} b\right)\left(\widetilde{F}_{n} c\right)\right],
$$

using the identity

$$
\widetilde{F}_{n}=\left(\widetilde{E}_{n+1}-\widetilde{E}_{n}\right)+\widetilde{F}_{n+1}
$$

Let the quantity in parentheses be denoted by $\widetilde{F}_{n, 1}$. The expansion generates eight terms. The term

$$
\widetilde{\operatorname{cum}}_{n, 1}(a, b, c)=\widetilde{E}_{n}\left[\left(\widetilde{F}_{n, 1} a\right)\left(\widetilde{F}_{n, 1} b\right)\left(\widetilde{F}_{n, 1} c\right)\right]
$$

from which $\widetilde{F}_{n+1}$ is absent is the leading order of the expansion. The seven terms with one, two or three $\widetilde{F}_{n+1}$ operators constitute the remainder term, $\widetilde{R}_{n, 1}(a, b, c)$. In fact, the terms in $\widetilde{R}_{n, 1}(a, b, c)$ with one $\widetilde{F}_{n+1}$ are zero as they each involve a fluctuation averaged against the product of two constant states; thus there are four non-zero remainder terms.

To continue this expansion of $\widetilde{a^{\prime \prime} b^{\prime \prime} c^{\prime \prime}}$, we employ the operator identity

$$
\widetilde{F}_{n}=\left(\widetilde{E}_{n+J+1}-\widetilde{E}_{n}\right)+\widetilde{F}_{n+J+1} .
$$

With the quantity in parentheses in the preceding equation denoted by $\widetilde{F}_{n, J}$,

$$
\widetilde{\operatorname{cum}}_{n}(a, b, c)=\widetilde{E}_{n}\left[\left(\widetilde{F}_{n, J} a\right)\left(\widetilde{F}_{n, J} b\right)\left(\widetilde{F}_{n, J} c\right)\right]+\widetilde{R}_{n, J}(a, b, c),
$$

where the remainder $\widetilde{R}_{n, J}(a, b, c)$ comprises the four nonzero terms involving the operator $\widetilde{F}_{n+J+1}$. The first term in Eq. (4.13), which we denote by $\widetilde{\operatorname{cum}}_{n, J}(a, b, c)$, is resolved on the grid $\mathcal{M}_{n+J+1}$.

5. Modeling. The discretized system of conservation laws, Eqs. (2.2)-(2.4), involves the SGS flux, defined by Eq. (2.3), which depends in an essential way on the solution $U$, not solely on the face average $\bar{U}=E_{n} U$. To close the governing system of equations, each SGS term must be related to the face average of the solution through a closure relation. In dynamic SGS modeling, the closure relation is determined with the aid of the Leonard SGS term.

5.1. Dynamic SGS modeling. We assume that the replacement for the SGS term for the grid level $\mathcal{M}_{n}$ takes the form $c_{n} M_{n}$ for some coefficient sequence $c_{n}$. Here $M_{n}$, is called the model for the particular SGS term. It is required to be a function of the cell averages of the primitive quantities in the dynamic equations, so that the 
expression for $M_{n}$ "closes". Furthermore, we make the asymptotic assumption that $c_{n}$ converges as $n \rightarrow \infty$, so that $c_{n}$ is approximately independent of $n$ when $n$ is large. The limit, denoted $c$, is the turbulent transport coefficient. For instance, when the SGS term is $\widetilde{\operatorname{cov}}_{n}(a, b)$, the closure, or modeling, relation is

$$
\widetilde{\operatorname{cov}}_{n}(a, b) \approx c M_{n} \text {. }
$$

If $\widetilde{\operatorname{cov}}_{n}(a, b)$ is a tensor quantity, so is the corresponding model $M_{n}$, and distinct coefficients are used for the deviatoric and spherical parts of this relationship.

Determination of the turbulent transport coefficient $c$ (LES step 5 in 1.4) proceeds as follows. Because the same coefficient $c$ relates the SGS term to the model at both grid levels $\mathcal{M}_{n-1}$ and $\mathcal{M}_{n}$, the difference $M_{n-1}-\widetilde{E}_{n-1} M_{n}$ is a model for the Leonard SGS term $\widetilde{\mathcal{L}}_{n-1}(a, b)$. We therefore compute $c$ by requiring that

$$
\widetilde{\mathcal{L}}_{n-1}(a, b) \approx c\left(M_{n-1}-\widetilde{E}_{n-1} M_{n}\right) .
$$

All expressions in Eq. (5.2), other than $c$, are $\mathcal{M}_{n}$-resolved. Thus, using (5.2), $c$ is also. For tensor SGS terms, $c$ is a scalar and (5.2) is interpreted in the sense of least squares.

The cancellation of terms in $\widetilde{\mathcal{L}}_{n-1}(a, b)$ is exact, whereas the assumption that $\widetilde{\operatorname{cov}}_{n}(a, b)$ is proportional to the model $M_{n}$ is an ansatz or approximation. The choice of model remains open for experimentation and improvement. Once the model has been specified, Eq. (5.2) determines the coefficient $c$ dynamically from the solution.

In keeping with the discussion in $\$ 1.4$ regarding choice of renormalization length scales, the turbulent transport coefficients of all SGS terms, excepting the anisotropic viscous term, are taken to be proportional to the anisotropic turbulent viscosity, with a dimensionless coefficient of proportionality.

5.2. Third-order cumulants and higher order expansion terms. We believe the closed form expansion terms derived here for the unclosed SGS terms will aid in future efforts to improve modeling of closure terms.

6. The Leading Order RNG Expansion. To leading order, we consider a single RNG step. With the current grid level denoted $\mathcal{M}=\mathcal{M}_{n}$, we also consider the once refined grid $\mathcal{M}_{n+1}$, for which each cell has been refined once in each mesh direction. On the cell faces of $\mathcal{M}_{n}$, we consider functions which are piecewise constant on cell faces of $\mathcal{M}_{n+1}$. In all cases, the leading order contribution to the unclosed SGS term is a product of two or more differences of primitive variables multiplied by an expression depending on $\rho$ and perhaps other variables which are not differenced. The differences occur in one or both of the directions tangential to the face of the grid cell. See A.11, A.16).

Detailed evaluation of these terms to offer possible improvement on SGS modeling will be the subject of a future publication.

7. Selection of SGS Terms. This section brings together the major themes of this paper: Nonuniqueness for solutions of a dynamic evolution problem, whether to use or omit SGS terms, their selection, if to be used, the role of the RNG as framework for understanding the selection of SGS terms, and the verification and validation $(V \& V)$ of a specific recipe (dynamic SGS) for the selection of SGS terms.

Without question, turbulence and turbulent mixing are important problems for scientific computing, which plays a central role in modern engineering design. Validation of these simulations (comparison to experiment) is an essential part of the 
scientific method. With nonunique and algorithmically dependent solutions, validation is hardly possible and instead, robust engineering design relies on calibration, which depends intrinsically on a sufficient range of experiments in a neighborhood of a design point.

We see a tight linkage between SGS terms and nonuniqueness. Solutions are observed to vary as a consequence of variation of the SGS terms. Omission of these terms, and their replacement by algorithmic artifacts also leads to a variation in the solution, i.e., nonuniqueness. Thus we find that specification of the SGS terms (in combination with some control over the numerical artifacts, primarily numerical diffusion) removes the nonuniqueness; nonuniqueness thus has its ultimate origin in underspecification of fluid transport. In the present case, with molecular transport added where convenient or necessary according to the laws of physics, it is the underspecified turbulent transport which gives rise to nonuniqueness of solutions, and the SGS terms, which specify subgrid turbulent transport, restore uniqueness. The RNG provides a framework for understanding this range of issues. Finally, having understood the problem, the dynamic selection of SGS terms provides a unique recipe for their selection. It then remains to show that this choice (dynamic SGS terms and control over numerical mass diffusion) satisfies the standards of V\&V.

7.1. Nonuniqueness of Solutions. Nonuniqueness is a strictly mathematical verification issue, which we address with equations specified at the continuum level of physics, ignoring considerations of kinetic theory and atomic length scales. We distinguish between macro and micro nonuniqueness. The distinction has to do with the observable used to measure nonuniqueness and its length scale in relation to $\Delta x$, in the limit $\Delta x \rightarrow 0$. An observable $\mathcal{O}$ (such as the RT instability growth rate $\alpha$ ) is called macro if its associated length scale $l_{\mathcal{O}}$ satisfies $l_{\mathcal{O}} \gg \Delta x$ and it is called micro (such as atomic mixing properties of the flow) if $l_{\mathcal{O}} \leq \Delta x$. We distinguish between mathematical nonuniqueness of the infinite Reynolds number Euler equations and the nonuniqueness of apparently converged LES simulations. We call the latter apparent LES nonuniqueness.

7.1.1. Examples of Nonuniqueness for Equations of Evolution. Mathematical nonuniqueness for solutions of the Euler equations is known [37, 7, 8. An understanding of the nature and origin of this nonuniqueness is presented in the survey [9. It is an open problem to determine the relevance to physics, if any, of the mathematical nonuniqueness theories. No features of these non-unique solutions are known which disqualify them from the point of view of either mathematics or physics. The strong convergence [4 of Navier-Stokes solutions to the Euler limit, even if extended conceptually to compressible flows, does not address uniqueness, and cannot, in so far as nonuniqueness is known mathematically.

Turning to physically relevant nonuniqueness, turbulent mixing is not the first time in which nonuniqueness has been an essential feature of a mathematical model of time dependent physical phenomena. Shock refraction problems, which describe self similar time dependent solutions, sometimes have multiple solutions. We note the nonuniqueness of the flame speed when analyzed at the level of the Euler equations. This nonuniqueness is removed by consideration of the relation between viscosity and thermal diffusion, in dimensionless terms the Prandtl number. Detonation waves exhibit multiple solutions, denote as weak or strong detonations [6]. Often some dissipative mechanism serves to select the weak detonation. Equations suggested by three phase flow models for petroleum reservoirs also show nonuniqueness for initial value problems associated with wave interactions (Riemann problems) [1, 2]. Within the 
TABLE 7.1

Variabilities in $\alpha$ from a variety of experimental and numerical sources

\begin{tabular}{|l|c|}
\hline \multicolumn{2}{|c|}{ Experimental variabilities } \\
\hline Experimental variability & $20 \%$ \\
Due to experimental initial conditions & $5-30 \%$ \\
\hline \multicolumn{2}{|c|}{ Numerical issues } \\
\hline ILES to experiment discrepancy [10] & $100 \%$ \\
ILES to ILES simulation discrepancy [10] & $50 \%$ \\
Numerical variation from transport coefficients [22, 23] & $5 \%$ \\
FT/LES/SGS to experiment discrepancy [23] & $5 \%$ \\
\hline
\end{tabular}

study of turbulence, the sensitivity of solutions to turbulence models is ubiquitous. We have thus noted noted precedents for nonuniqueness of solutions to time dependent problems when analyzed at the Euler level. This ambiguity is often removed when the modeled at the Navier-Stokes level. However, for turbulence modeling, the analogous resolution of ambiguity requires specifying the turbulent transport, exactly the quantity which introduces the ambiguity. Nonuniqueness of apparently converged LES is observed in practice and is an issue that solution verification methodology has yet to address [29].

7.1.2. Theoretically Inferred Nonuniquenes. The conceptual analysis of cases A and B of 1.2 suggests nonuniqueness parametrized by points of the space $\mathcal{T}$ of dimensionless total (molecular plus turbulent) transport.

7.1.3. Numerically Observed Nonuniqueness. A wide range of simulation results have been proposed as solutions for an identical high Re turbulent mixing problem [10. Code comparison [27] of apparently converged solutions showed differences in thermal mixing properties. Additionally, systematic variation of the SGS coefficient has been observed to change the atomic mixing properties of nominally converged solutions [29]. On this basis, we consider here the extent to which the selection of the high Re limit might be influenced by numerical considerations. Consistent with this point of view are comments from [15]: "Results using the MILES approach for LES are found to strongly depend on scheme parameters and grid size. Also, physical variables cannot be simultaneously predicted." See also [39, 11, 3]. In Table 7.1, we summarize the observed numerical and experimental variation in efforts to determine the overall growth rate of the Rayleigh-Taylor instability, known as $\alpha$. As a contrast to the above ILES picture, we note the good validation results which have been achieved using FT/LES/SGS (see Tables 7.1 and 7.2) also using DNS [33. The experiments RT give unique results, i.e., they are repeatable (we allocate the $5-30 \%$ experimental variation in RT $\alpha$ predominantly to variation in recorded experimental conditions, in keeping with our simulations which duplicate the experiment to experiment variation in $\alpha$ ), so that any solution (such as the FT/LES/SGS solution) which agrees with these experiments is itself numerically unique.

7.1.4. The RNG Framework and Nonuniqueness. The basic ideas relating the RNG framework to nonuniqueness are explained in Sec. 1. Relevant to nonuniqueness is the key RNG step of setting the parameters for the essential variables. (See Sec. 1.3.) We identify the essential variables, tentatively and as a scientific judgment, as the turbulent transport coefficients. These are set in RNG methodolgy by comparison to experiment, and here, in lieu of an experiment, as a coefficient of a model 
TABLE 7.2

Comparison of FT simulation to experiment. Discrepancy refers to the comparison of results outside of uncertainty intervals, if any, as reported.

\begin{tabular}{|ccc|cc|c|}
\hline Ref. & Exp. & Sim. Ref. & $\alpha_{\exp }$ & $\alpha_{\text {sim }}$ & Discrepancy \\
\hline$[40]$ & $\# 112$ & {$[22]$} & 0.052 & 0.055 & $6 \%$ \\
{$[40]$} & $\# 105$ & {$[14$} & 0.072 & $0.076 \pm 0.004$ & $0 \%$ \\
{$[40,36]$} & 10 exp. & {$[12$} & $0.055-0.077$ & 0.066 & $0 \%$ \\
{$[35$} & air-He & {$[25]$} & $0.065-0.07$ & 0.069 & $0 \%$ \\
{$[34]$} & Hot-cold & {$[22,[14$} & $0.070 \pm 0.011$ & 0.075 & $0 \%$ \\
{$[34$} & Salt-fresh & {$[14$} & $0.085 \pm 0.005$ & 0.084 & $0 \%$ \\
\hline
\end{tabular}

(Sec. 1.4). For the dynamic choice of SGS terms, the coefficient is determined from the simulation itself in Sec. 5.1. The simulation is thus parameter free, and nonuniqueness has been removed. The nonuniqueness originated in the unspecified turbulent transport and was removed by the RNG setting of the coefficients of the essential variables, with the setting determined by the dynamic method and thus uniquely.

7.2. Verification and Validation. In [29, we outline a validation/verification program for LES in the high Re regime, based on the code FT/LES/SGS. The LES/SGS framework has no adjustable parameters. Our validation is in the RT experimental regime. We have validated the FT/LES/SGS code by comparison to experimental measurements of the RT growth rate $\alpha$, conducted at $\operatorname{Re} \sim 3.5 \times 10^{4}$. This validation tests the transport coefficients, which in the LES/SGS framework, are not adjustable, and which are much more sensitive in the RT experiments than in the RM experiments. Additionally, through code comparison (referred to above), we compare FT to RAGE on RM problems and rely on the RM validation of RAGE. Beyond the experimental Re range of $3.5 \times 10^{4}$, we employ an extrapolation, ie a mathematical verification step, to Re values of $6 \times 10^{5}$ to $6 \times 10^{7}$ or higher. We observe that the transport coefficients and the atomic level mixing CDFs display only a mild norm dependence (a 10\% to 15\% effect) resulting from a change in the values of Re and are also a norm convergence under mesh refinement, in a purely hydro study.

7.2.1. V\&V for RT and RM Instabilities. We have conducted extensive studies of RT instabilities, as V\&V for the FT/LES/SGS algorithm. Expanding on the bottom two lines of Table 7.1, we summarize the principal results in Table 7.2 . We observe nearly perfect agreement between simulation and experiment, within error bars, and accuracy sufficient to distinguish between the distinct $\alpha$ s of distinct experiments. In this way, we show that the variation in $\alpha$ across multiple experiments is caused partly by initial conditions (the water channel splitter plate experiments introduce significant noise) and partly by changes in the fluid transport properties of the fluids [22, 23. The common belief that significant long wave length noise present in the initial data explains the factor of two discrepancy between experiment and the numerous ILES simulations, has been shown to be false [14. The effect on $\alpha$ from the long wave length perturbations in the initial condition of [40] was shown [14] to be $\pm 5 \%$.

$\mathrm{V} \& \mathrm{~V}$ for micro observables is still an open research question, but partial results have been obtained. Convergence properties of the second moments of concentration and the CDF (cumulative distribution function) for a RT instability are analyzed in 16, 18, 24. Convergence of the CDFs for the joint concentration-temperature (micro) variables of an RM instability were studied in [29, 30. 
7.2.2. Comparison of Distinct SGS Model Formulations. The primary comparison issue addressed in this paper is the comparison of a dynamic SGS algorithm, mostly of FT/LES/SGS to ILES, based on published studies of RT simulations by the ILES method, as discussed in Sec. 7.1.3. The issue here is whether to use SGS terms or not, whether they should be explicitly identified as part of the simulation package or located implicitly within the algorithm itself in a non-transparent manner. Among the problems with ILES, and perhaps related to its performance problems evident from Table 7.1 of Sec. 7.1.3 is the fact that the dimensionless turbulent coefficients are ratios. Algorithms which seek to optimize (and perhaps even succeed in optimizing) the numerator and the denominator of a ratio separately can still fail dramatically in optimizing the ratio. Conceptually, the basis for optimization of the numerator and the denominator is to reduce their size. But there is no conceptual basis for optimization of the ratio. To the extent that separate optimization of the numerator and denominator succeeds, the ratio is the indeterminant expression $0 / 0$. It would seem that the ILES method, as it is generally explained, does not offer a plan for such an optimization.

The methods used in the FT/LES/SGS algorithm are a variant of the standard dynamic SGS models, in that we average over a single mesh block, rather than use an a localizing averaging kernel (filter), conventionally extending over five mesh blocks. Additionally, in the numerical implementation, the cell face averages are replaced by cell centered quantities, an approximation which reduces the accuracy of these terms to first order. We do not expect a major difference between the cell block averages used here (called an implicit filter) and the conventional extended filter for the dynamic SGS method. Aside from the simpler conceptual analysis of the mesh block averages, a feature exploited in the present paper, we prefer the mesh block averages as this method appears to be favorable when coupling to reactive flows (turbulent combustion), with finite rate chemistry. An assessment of this proposal will appear at a later time.

A separate and important question, but out of the scope of this paper, is to a quantitative comparison of the variety of turbulence algorithms which do include explicit SGS terms. The reader is referred to a variety of survey and review documents e..g., 32, 20, for this active research topic. Dynamic SGS models [21, 31] are arguably the standard method to which others are compared. We also mention the approximate deconvolution method (ADM), with a recent contribution [38. Comparisons involve specific flows and specific features of these flows. In such comparisons, sometimes alternate methods excel. Classical problems of turbulent flow fall into this category, such as wall bounded turbulence, turbulent shock boundary layer interactions, turbulent combustion, and turbulent particulate flow. Complex flows are an open research area, for which a universal solution appears unlikely.

8. Discussion. We have re-examined the relation between LES and the RNG. LES cannot be an RNG fixed point as it fails to be scale invariant. However, the RNG expansion still applies, and based on this, we derive a closed form expansion for the unclosed SGS terms. To leading order, this expansion coincides with the Leonard stress in the derivation of the dynamic SGS models. The full expansion of the unclosed terms suggests a higher order determination of the model coefficients. For design of aircraft and for flow in pipes, lift and drag, the important observables, are macro in nature. For reactive flows, such as combustion, micro observables of atomic mixing properties are fundamental. They are the direct input to continuum level chemical reaction rate laws. Accurate modeling of micro observables allows finite 
rate LES chemistry, and elimination of flame structure models from combustion simulations. Practical requirements of engineering simulations also lead us to emphasize the importance of LES and of compressible simulations.

Apparent LES nonuniqueness (both macro and micro) is known, as reviewed in 97 The macro level apparent LES nonuniqueness of RT simulations speaks for itself, even if its mathematical status in not known. It seems safe to speculate that the apparent LES nonuniqueness at the micro (as opposed to the macro) level, is more likely to survive future levels of mesh refinement and careful control over simulation input data, and in this sense it may be scientifically more fundamental.

We discuss the possible role of numerical and physical modeling issues in the selection of a high Re limit point. We have identified the RNG relevant variables as the dimensionless parameters of the Euler equation. The identification of these parameters as relevant is conventional within RNG methods, but it is neither demonstrated by the results of this paper nor is it essential to them. RNG theories may also include dimensional equation parameters, as with the mass of the electron in quantum field theory. Some of the dimensionless turbulent transport parameters could turn out to be irrelevant (decreasing more rapidly under RNG iterations) and still parametrize non-unique solutions, to be achieved by a stronger fractal or numerical algorithmic forcing.

There can be little doubt that a scientific understanding of turbulent mixing and its dependence on Re in the simple "pure hydro" example considered here provides an indispensable foundation for the study of mixing in the multi-physics context likely to prevail in complex problems of engineering interest.

\section{Appendix A. Elementary Formulas.}

Here we gather some elementary formulas, derived in detail for the convenience of the reader.

A.1. Covariance. We begin with the two-dimensional $(D=2)$ case of the grid $\mathcal{M}_{1}$, which subdivides $[0,1] \times[0,1]$ into four squares, and a particular face $f$ of the grid $\mathcal{M}_{0}$, say $[0,1] \times\{0\}$, which is subdivided by $\mathcal{M}_{1}$ into two segments. A quantity $a=$ $E_{1} a$ that is $\mathcal{M}_{1}$-resolved takes on a constant value on each of the two segments of $f$; we represent $a$ as the two-dimensional vector $a=\left(a_{1}, a_{2}\right)^{T}$. Such vectors form a twodimensional real Hilbert space, provided we adopt an inner product. For the standard inner product $\langle\cdot, \cdot\rangle$, the projection $E_{0}$ onto constant vectors and the complementary projection $F_{0}=I-E_{0}$ are given by

$$
E_{0}=\frac{1}{2}\left(\begin{array}{ll}
1 & 1 \\
1 & 1
\end{array}\right), \quad F_{0}=\frac{1}{2}\left(\begin{array}{rr}
1 & -1 \\
-1 & 1
\end{array}\right)
$$

Now let $\rho=\left(\begin{array}{cc}\rho_{1} & 0 \\ 0 & \rho_{2}\end{array}\right)$, where $\rho_{1}, \rho_{2}>0$, and consider the two-dimensional real Hilbert space with weighted inner product $\langle\cdot, \cdot\rangle_{\rho}$ defined by

$$
\langle a, b\rangle_{\rho}=\rho_{1} a_{1} b_{1}+\rho_{2} a_{2} b_{2}=\langle a, \rho b\rangle
$$

for vectors $a$ and $b$. Next we compute the adjoint $A^{* \rho}$ of a matrix $A=\left(\begin{array}{cc}a_{11} & a_{12} \\ a_{21} & a_{22}\end{array}\right)$ with respect to $\langle\cdot, \cdot\rangle_{\rho}$.

For vectors $a=\widetilde{E}_{1} a$ and $b=\widetilde{E}_{1} b$,

$$
\langle b, A a\rangle_{\rho}=\langle b, \rho A a\rangle=\left\langle b, \rho A \rho^{-1} \rho a\right\rangle=\left\langle\rho^{-1} A^{T} \rho b, \rho a\right\rangle=\left\langle\rho^{-1} A^{T} \rho b, a\right\rangle_{\rho},
$$


where $A^{T}$ is the adjoint with respect to the usual inner product, i.e., the transpose of $A$. Thus

$$
A^{* \rho}=\rho^{-1} A^{T} \rho=\left(\begin{array}{cc}
a_{11} & \left(\rho_{2} / \rho_{1}\right) a_{21} \\
\left(\rho_{1} / \rho_{2}\right) a_{12} & a_{22}
\end{array}\right) .
$$

Therefore $A$ is self-adjoint in the $\rho$ inner product if and only if $a_{12}=\left(\rho_{2} / \rho_{1}\right) a_{21}$.

Having determined the adjoint operation, we verify that

$$
\begin{aligned}
& \widetilde{E}_{0}=\frac{1}{\rho_{1}+\rho_{2}}\left(\begin{array}{cc}
\rho_{1} & \rho_{2} \\
\rho_{1} & \rho_{2}
\end{array}\right)=\frac{1}{\rho_{1}+\rho_{2}}\left(\begin{array}{l}
1 \\
1
\end{array}\right)\left(\begin{array}{ll}
\rho_{1} & \rho_{2}
\end{array}\right), \\
& \widetilde{F}_{0}=\frac{1}{\rho_{1}+\rho_{2}}\left(\begin{array}{cc}
\rho_{2} & -\rho_{2} \\
-\rho_{1} & \rho_{1}
\end{array}\right)=\frac{1}{\rho_{1}+\rho_{2}}\left(\begin{array}{c}
-\rho_{2} \\
\rho_{1}
\end{array}\right)\left(\begin{array}{ll}
-1 & 1
\end{array}\right)
\end{aligned}
$$

are the self-adjoint projection operators onto the subspace of constant vectors and its complement with respect to $\langle\cdot, \cdot\rangle_{\rho}$, the latter comprising states $\rho$-orthogonal to constant vectors.

With these preparations, we compute that

$$
\left\langle b, \widetilde{E}_{0} a\right\rangle_{\rho}=\left\langle\left(\begin{array}{l}
b_{1} \\
b_{2}
\end{array}\right), \frac{\rho_{1} a_{1}+\rho_{2} a_{2}}{\rho_{1}+\rho_{2}}\left(\begin{array}{l}
1 \\
1
\end{array}\right)\right\rangle_{\rho}=\frac{\left(\rho_{1} b_{1}+\rho_{2} b_{2}\right)\left(\rho_{1} a_{1}+\rho_{2} a_{2}\right)}{\rho_{1}+\rho_{2}}
$$

and

$$
\left\langle b, \widetilde{F}_{0} a\right\rangle_{\rho}=\left\langle\left(\begin{array}{l}
b_{1} \\
b_{2}
\end{array}\right), \frac{a_{2}-a_{1}}{\rho_{1}+\rho_{2}}\left(\begin{array}{c}
-\rho_{2} \\
\rho_{1}
\end{array}\right)\right\rangle_{\rho}=\frac{\rho_{1} \rho_{2}}{\rho_{1}+\rho_{2}}\left(b_{2}-b_{1}\right)\left(a_{2}-a_{1}\right) .
$$

Notice that

$$
\left\langle b, \widetilde{F}_{0} a\right\rangle_{\rho}=\left\langle\widetilde{E}_{1} b, \widetilde{F}_{0} \widetilde{E}_{1} a\right\rangle_{\rho}=\left\langle b, \widetilde{E}_{1} \widetilde{F}_{0} a\right\rangle_{\rho}
$$

Therefore the expression $\widetilde{E}_{0}\left[b \widetilde{E}_{1} \widetilde{F}_{0} a\right]$, which is the leading order term in the expansion (4.8) of $\widetilde{a^{\prime \prime} b^{\prime \prime}}$ for $n=0$, takes the value (A.8) on the face $f$ of $\mathcal{M}_{0}$. This value is the product of finite differences.

We extend this analysis to three dimensions $(D=3)$ for a face $f$ of an elementary $2^{3}$ grid. The face is divided into a $2 \times 2$ grid with, say, tangential coordinate directions 1 and 2 . The four values of a quantity $a$ on $f$ constitute the vector $\left(a_{11}, a_{12}, a_{21}, a_{22}\right)^{T}$. In terms of this notation, the $\rho$-orthogonal projection onto constant vectors is

$$
\widetilde{E}_{0}=\frac{1}{\rho_{11}+\rho_{12}+\rho_{21}+\rho_{22}}\left(\begin{array}{l}
1 \\
1 \\
1 \\
1
\end{array}\right)\left(\begin{array}{llll}
\rho_{11} & \rho_{12} & \rho_{21} & \rho_{22}
\end{array}\right) .
$$

Explicit calculation then shows that

$$
\left\langle b, \widetilde{F}_{0} a\right\rangle_{\rho}=\left(\sum_{i, j} \rho_{i j}\right)^{-1} \sum_{(i, j)<(k, \ell)} \rho_{i j} \rho_{k \ell}\left(b_{k l}-b_{i j}\right)\left(a_{k l}-a_{i j}\right),
$$

where index pairs are ordered lexicographically, so that there are six terms in the sum. Each term is again the product of finite differences. 
A.2. Third-order cumulants. To study the third-order cumulant, we examine the inner products

$$
\left\langle a,\left(\widetilde{F_{0}} b\right)\left(\widetilde{F_{0}} c\right)\right\rangle_{\rho} \quad \text { and } \quad\left\langle\widetilde{F_{0}} a,\left(\widetilde{F_{0}} b\right)\left(\widetilde{F_{0}} c\right)\right\rangle_{\rho},
$$

first for a single face in the $D=2, \mathcal{M}_{1}$ context. By (A.8), we have

$$
\widetilde{F_{0}} a=\frac{a_{2}-a_{1}}{\rho_{1}+\rho_{2}}\left(\begin{array}{c}
-\rho_{2} \\
\rho_{1}
\end{array}\right) \text {. }
$$

Thus

$$
\left(\widetilde{F_{0}} b\right)\left(\widetilde{F_{0}} c\right)=\frac{\left(b_{2}-b_{1}\right)\left(c_{2}-c_{1}\right)}{\left(\rho_{1}+\rho_{2}\right)^{2}}\left(\begin{array}{c}
\rho_{2}^{2} \\
\rho_{1}^{2}
\end{array}\right)
$$

and

$$
\begin{array}{r}
\left\langle a,\left(\widetilde{F_{0}} b\right)\left(\widetilde{F_{0}} c\right)\right\rangle_{\rho}=\left(a_{1} \rho_{1}+a_{2} \rho_{2}\right) \frac{\rho_{1} \rho_{2}}{\rho_{1}+\rho_{2}}\left(b_{2}-b_{1}\right)\left(c_{2}-c_{1}\right), \\
\left\langle\widetilde{F_{0}} a,\left(\widetilde{F_{0}} b\right)\left(\widetilde{F_{0}} c\right)\right\rangle_{\rho}=\left(a_{2}-a_{1}\right)\left(b_{2}-b_{1}\right)\left(c_{2}-c_{1}\right) \frac{\rho_{1} \rho_{2}\left(\rho_{2}-\rho_{1}\right)}{\left(\rho_{1}+\rho_{2}\right)^{2}} .
\end{array}
$$

Again we extend this analysis to three dimension, for a face of an elementary $2^{3}$ grid. Consider a face parallel to the $x, y$ plane. We introduce the $x$ direction average operator $E_{0, x}$, which projects onto states constant in the $x$ direction, and its orthogonal compliment, $F_{0, x}$, which projects onto states orthogonal to $x$ direction constants. Similarly we introduce $E_{0, y}$ and $F_{0, y}$. We have

$$
I=E_{0, x}+F_{0, x}=E_{0, x} E_{0, y}+E_{0, x} F_{0, y}+F_{0, x} E_{0, y}+F_{0, x} F_{0, y} .
$$

We substitute this identity into the cumulant expression, in front of each of $a, b, c$. Only terms with at least on $F$ for each factor are nonzero, and so there are 27 of these. We do not present detailed formulas for the 27 terms, but we note that each $F$ introduces a first difference operator. Thus each of the 27 terms is a monomial in first differences applied at least once to each of $a, b, c$. It has cubic, up to 6 power of first differences within the 27 terms.

\section{REFERENCES}

[1] A. Azevedo And D. Marchesin, Multiple viscous solutions for systems of conservation laws, Trans. Amer. Math. Soc., 347 (1995), pp. 3061-3078.

[2] A. Azevedo, D. Marchesin, B. Plohr, and K. Zumbrun, Nonuniqueness of nonclassical solutions of Riemann problems, Zeit. angew. Math. Phys., 47 (1996), pp. 977-998.

[3] B. Camguilhem, A. Honein, And P. Moin, Evaluation of a Monotone Integrated Large Eddy Simulation Algorithm, in APS Division of Fluid Dynamics Meeting Abstracts, Nov. 2002, p. J5.

[4] G.-Q. Chen And J. Glimm, Kolmogorov's theory of turbulence and inviscid limit of the NavierStokes equations in $R^{3}$, Commun. Math. Phys., 310 (2012), pp. 267-283.

[5] R. A. Clark and J. H. Ferziger abd W. C. Reynolds, Evaluation of subgrid-scale models using accurately simulated turbulent flows, J. Fluid Mech., 91 (1979).

[6] R. Courant and K. Friedrichs, Supersonic Flow and Shock Waves, Springer-Verlag, New York, 1967.

[7] C. De Leliss and L. Szekelyhidi, The Euler equations as a differential inclusion, Ann. Math., 170 (2009), pp. 1471-1436. 
[8] — On admissibility criteria for weak solutions of the Euler equations, Arch. Rat. Mech. Anal., 195 (2010), pp. 225-260.

[9] - The h-principle and the equations of fluid dynamics, Bull. Amer. Math. Soc., 49 (2012), pp. 347-376.

[10] G. Dimonte, D. L. Youngs, A. Dimits, S. Weber, M. Marinak, S. Wunsch, C. Garsi, A. Robinson, M. Andrews, P. Ramaprabhu, A. C. Calder, B. Fryxell, J. Bielle, L. Dursi, P. Macniece, K. Olson, P. Ricker, R. Rosner, F. Timmes, H. Tubo, Y.-N. Young, AND M. Zingale, A comparative study of the turbulent Rayleigh-Taylor instability using high-resolution three-dimensional numerical simulations: The Alpha-Group collaboration, Phys. Fluids, 16 (2004), pp. 1668-1693.

[11] E. Garnier, M. Mossi, P. Sagaut, P. Comte, and M. Delville, On the use of shockcapturing schemes for large-eddy simulation, J. Comput. Phys., 153 (1999), p. 273.

[12] E. George, J. Glimm, X.-L. Li, Y.-H. Li, And X.-F. Liu, The influence of scale-breaking phenomena on turbulent mixing rates, Phys. Rev. E, 73 (2006), p. 016304.

[13] M. Germano, Tech. Report 116, Center for Turbulence Research, Stanford University and NASA-Ames Research Center, 1990.

[14] J. Glimm, D. H. Sharp, T. Kaman, and H. Lim, New directions for Rayleigh-Taylor mixing, Phil. Trans. Royal Soc. A: Turbulent mixing and beyond, (2012). in press. Los Alamos National Laboratory Preprint LA-UR 11-00423 and Stony Brook University Preprint SUNYSB-AMS-11-01.

[15] A. Honein And P. Moin, Numerical aspects of compressible turbulence simulations, Report No. TF-92, Stanford Univ., 2005.

[16] T. Kaman, R. Kaufman, J. Glimm, and D. H. Sharp, Uncertainty quantification for turbulent mixing flows: Rayleigh-Taylor instability, in Uncertainty Quantification in Scientific Computing, A. Dienstfrey and R. Boisvert, eds., vol. 377 of IFIP Advances in Information and Communication Technology, Springer, 2012, pp. 212-225. Stony Brook University Preprint number SUNYSB-AMS-11-08.

[17] T. Kaman, H. Lim, Y. Yu, D. Wang, Y. Hu, J.-D. Kim, Y. Li, L. Wu, J. Glimm, X. JiaO, X.L. Li, AND R. SAmulyak, A numerical method for the simulation of turbulent mixing and its basis in mathematical theory, in Lecture Notes on Numerical Methods for Hyperbolic Equations: Theory and Applications: Short Course Book, CRC/Balkema, London, 2011, pp. 105-129. Stony Brook University Preprint SUNYSB-AMS-11-02.

[18] Ryan Kaufman, Tulin Kaman, Yan Yu, and James Glimm, Stochastic convergence and the software tool $W^{*}$, in Proceeding Book of International Conference to honour Professor E.F. Toro, CRC, Taylor and Francis Group, 2012, pp. 37-41. Stony Brook University Preprint number SUNYSB-AMS-11-10.

[19] A. N. Kolmogorov, Local structure of turbulence in incompressible viscous fluid for very large Reynolds number, Doklady Akad. Nauk. SSSR, 30 (1941), pp. 299-3031.

[20] Eric Lamballais, Rainer Friedrich, Bernard J. Geurts, and Olivier Metais, eds., Direct and Large Eddy Simulation VI, Springer Verlag, Heidelberg, 2006.

[21] A. LEONARD, On the energy cascade in large-eddy simulations of turbulent fluid flows, Adv. Geophys. A, 18 (1974), pp. 237-248.

[22] H. Lim, J. Iwerks, J. Glimm, And D. H. Sharp, Nonideal Rayleigh-Taylor mixing, Proc. Nat. Acad. Sci., 107(29) (2010), pp. 12786-12792. Stony Brook University Preprint SUNYSBAMS-09-05 and Los Alamos National Laboratory Preprint LA-UR 09-06333.

[23] H. Lim, J. Iwerks, Y. YU, J. Glimm, And D. H. Sharp, Verification and validation of a method for the simulation of turbulent mixing, Physica Scripta, T142 (2010), p. 014014. Stony Brook University Preprint SUNYSB-AMS-09-07 and Los Alamos National Laboratory Preprint LA-UR 09-07240.

[24] H. Lim, T. Kaman, Y. Yu, V. Mahadeo, Y. Xu, H. Zhang, J. Glimm, S. Dutta, D. H. Sharp, AND B. Plohr, A mathematical theory for LES convergence, Acta Mathematica Scientia, 32 (2012), pp. 237-258. Stony Brook University Preprint SUNYSB-AMS-11-07 and Los Alamos National Laboratory Preprint LA-UR 11-05862.

[25] X.-F. Liu, E. George, W. Bo, And J. Glimm, Turbulent mixing with physical mass diffusion, Phys. Rev. E, 73 (2006), p. 056301.

[26] T. MA, Large eddy simulation of variable density flows, PhD thesis, University of Maryland, 2006.

[27] T. O. MAsser, Breaking Temperature Equilibrium in Mixed Cell Hydrodynamics, PhD thesis, State University of New York at Stony Brook, 2007.

[28] W. D. МсСомв, Renormalization Methods, Oxford University Press, Oxford, 2004.

[29] J. Melvin, P. Rao, R. Kaufman, H. Lim, Y. Yu, J. Glimm, and D. H. Sharp, Atomic scale mixing for inertial confinement fusion associated hydro instabilities, High Energy Density 
Physics, (2013). In Press. Stony Brook University Preprint SUNYSB-AMS-12-01 and Los Alamos National Laboratory Preprint LA-UR 12-21555.

[30] - Turbulent transport at high reynolds numbers in an icf context, ASME Journal of Fluids Engineering, submitted (2013).

[31] P. Moin, K. Squires, W. Cabot, and S. Lee, A dynamic subgrid-scale model for compressible turbulence and scalar transport, Phys. Fluids A, 3 (1991), pp. 2746-2757.

[32] C. Montveau and J. Katz, Scale invariance in large eddy simulation of turbulence, Ann Rev. Fluid Mech., 32 (2000), pp. 1-32.

[33] N. Mueschke And O. Schilling, Investigation of Rayleigh-Taylor turbulence and mixing using direct numerical simulation with experimentally measured initial conditions. I. Comparison to experimental data, Physics of Fluids, 21 (2009), pp. 014106 1-19.

[34] Nicholas J. MueschKe, Experimental and numerical study of molecular mixing dynamics in Rayleigh-Taylor unstable flows, PhD thesis, Texas A and M University, 2008.

[35] P. Ramaprabhu And M. Andrews, Experimental investigation of Rayleigh-Taylor mixing at small atwood numbers, J. Fluid Mech., 502 (2004), pp. 233-271.

[36] K. I. READ, Experimental investigation of turbulent mixing by Rayleigh-Taylor instability, Physica D, 12 (1984), pp. 45-58.

[37] V. SCHEFFER, An inviscid flow with compact support in space-time, J. Geom. Anal., 3 (1993), pp. 343-401.

[38] Phillpp Schlater, Steffan Soltz, and Leonard Kaiser, An approximate deconvolution procedure for large-eddy simulation, in Direct and Large Eddy Simulation VI, E. Lamballais, Reiner Freidrich, Bernard J. Geurts, and Olivier Metais, eds., Springer Verlag, Heidelberg, 2006, pp. 135-142.

[39] A. Silveira-Neto, D. Grand, O. Métais, and M. Lesieur, A numerical investigation of the coherent structures behind a backward-facing step, J. Fluid Mech., 256 (1993), pp. 1-25.

[40] V. S. Smeeton And D. L. Youngs, Experimental investigation of turbulent mixing by RayleighTaylor instability (part 3), AWE Report Number 0 35/87, 1987. 